\title{
The Experience of Work Engagement, Hardy Personality, Optimism and Subjective Well-Being among Nurses from China and Spain
}

\author{
Eva Garrosaํ, Felix Ladstätter², Bernardo Moreno', Yiqun Gan³ ${ }^{3}$ Isabel Carmona1 \\ ${ }^{1}$ Faculty of Psychology, UAM, Spain \\ ${ }^{2}$ Department of Psychology, IE University, Segovia, Spain \\ ${ }^{3}$ Department of Psychology, Beijing University, Beijing, China \\ Email: eva.garrosa@uam.es \\ Received January 2014
}

\begin{abstract}
Engaged nurses have a sense of energetic and effective connection with their work activities and they see themselves as fully capable of dealing with the demands of their job. To analyze the experience of work engagement, examining cross-national differences in Hardy Personality (HP), optimism and subjective well-being among nurses from China and Spain. Cross-cultural study with a sample with 154 nurses from Beijing (China) and 164 nurses from Madrid (Spain) who completed the Engagement, HP, Optimism and Subjective Well-Being (SWB) Scales. The data were analyzed using Pearson correlations, Student's t-tests and hierarchical multiple regression. The personality characteristics in these countries are different, as are the variables that explain engagement. In both samples, the variable most closely associated with engagement is commitment. Optimism was another predictor, but subjective well-being was only a predictor for Spanish nurses. This study is a first approximation to understand the application of HP, optimism and subjective well-being as personal resources that affect the way in which nurses interact with their working environment and to improve engagement. Practical implications are discussed.
\end{abstract}

\section{Keywords}

Cross-Cultural, Engagement, Hardy Personality, Nurses, Optimism, Subjective Well-Being

\section{Introduction}

Engaged employees have a sense of energetic and effective connection to their work activities and they see themselves as fully capable of dealing with the demands of their job. Thus, work engagement has important consequences for employee performance, organizational management and satisfaction. There is significant evidence of the relationship between employee engagement at work and organizational outcomes. Despite these 
positive consequences, engagement in nursing has not yet been sufficiently studied. Therefore, authors like Simpson [1] underscore the importance of our theoretical and practical understanding of nurse engagement in order to prioritize and implement interventions aimed at improving nurse performance, patient outcomes, work environment and mutual aid in the organization and other core healthcare organizational outcomes. The construct of engagement provides a more complex and thorough perspective of an individual's relationship with work. From this perspective, HP, optimism and SWB are relevant personal resources that affect the way people interact with their working environment. For instance, in the Job Demands-Resources Model [2], optimism, self-efficacy, and resilience have been shown to be personal resources related to work engagement. HP can be defined as hardy attitudes: the belief that one has control over or can influence events, feeling committed to activities, and having a good relationship with oneself-in the sense that one recognizes one's own distinctive values, goals and priorities in life-, and viewing change as a challenge rather than a threat. HP has an impact on the stress/health relationship and has been contrasted in the literature. There is also some evidence that optimism may be positively associated with affective measures of employee attitudes such as increased job satisfaction and organizational commitment. Likewise, workers' SWB seems to be related to commitment and enthusiasm for work. Particularly, the broaden-and-build theory suggests that positive affective states expand the behavioral repertoire, thus facilitating more efficacious responses and generating upward spirals of well-being. In the workplace context, various studies have suggested that happy people are more productive. The aim of this study was to analyze the experience of work engagement, examining cross-cultural differences in HP, optimism and SWB among nurses from China and Spain, as representing the collectivism/individualism dimensions proposed by Hofstede [3]. The present study tests the following hypotheses: Hypothesis 1. HP dimensions, optimism and SWB are positively related to work engagement (vigor, dedication and absorption) when the percentages of interaction with patients are taken into account in both samples. Hypothesis 2. Personality predictors of work engagement in China and Spain will show some cross-cultural differences between nurses from China and Spain.

\section{Method}

Cross-cultural study with a sample with 154 nurses from Beijing (China) and 164 nurses from Madrid (Spain) who completed the Engagement, HP, Optimism and SWB Scales. Inclusion criteria were working in a hospital and interacting with patients (providing patient care). A total of $90 \%$ of the participants were female and the other $10 \%$ were male, with a mean age of 33 years $(S D=10.86)$. All were full-time nurses and had an average of 12 years of experience $(\mathrm{SD}=8.8)$.

\section{Results}

To determine the effects of the predictors on the engagement dimensions, the percentage of interaction with the patients was entered first as a control variable, and then the HP components, optimism and SWB were sequentially entered in the model. The increase in $\mathrm{R}^{2}\left(\Delta \mathrm{R}^{2}\right)$ was calculated to determine the relative contributions of each set of variables. Standardized coefficients $(\beta)$ were calculated to compare the relative importance of each variable in the model. The data were checked for multicollinearity, using tolerance and the variance inflation factor (VIF). Values of VIF greater than 10 and tolerance-values smaller than 0.10 may indicate multicollinearity. There were no signs of multicollinearity in any of the six regression models. The analyses were performed with the SPSS-program (see Tables 1 and 2).

\section{Discussion}

Nurses are frequently found among the professionals who express a higher degree of dissatisfaction, and a large percentage of them wish to leave the profession in the next five years. We therefore need to investigate the elements that could slow down this negative tendency. Positive work processes such as dedication, absorption, and vigor are opposed to these negative feelings of dissatisfaction. As some aspects of engagement depend on concrete organizational characteristics, it is important for organizations to promote the development of nurses' hardiness and positive personality resources so they can manage the continuous contact with pain and suffering adaptively, and deal adequately with the negative emotions that emerge at work. Thus, the variables used in all the analyses with both samples explain a significant percentage of the scores in engagement. However, Hypo- 
Table 1. Results of hierarchical regression analyses, standardized regression coefficients. Personality predictors of work engagement among Chinese nurses.

\begin{tabular}{|c|c|c|c|c|c|c|c|c|c|c|c|c|}
\hline & \multicolumn{4}{|c|}{ Dedication } & \multicolumn{4}{|c|}{ Absorption } & \multicolumn{4}{|c|}{ Vigor } \\
\hline & Step 1 & Step 2 & Step 3 & Step 4 & Step 1 & Step 2 & Step 3 & Step 4 & Step 1 & Step 2 & Step 3 & Step 4 \\
\hline $\begin{array}{c}\% \text { of } \\
\text { interaction } \\
\text { with patients }\end{array}$ & $-0.21^{* *}$ & $-0.17^{* *}$ & $-0.17^{* *}$ & $-0.15^{* *}$ & $-0.23^{* *}$ & $-0.19^{* *}$ & $-0.19^{* *}$ & $-0.18^{* *}$ & $-0.29^{* * *}$ & $-0.24^{* * *}$ & $-0.24^{* * *}$ & $-0.23^{* *}$ \\
\hline Commitment & & $0.69^{* * *}$ & $0.66^{* * *}$ & $0.61^{* * *}$ & & $0.62^{* * *}$ & $0.57^{* * *}$ & $0.53^{* * *}$ & & $0.58^{* * *}$ & $0.54^{* * *}$ & $0.48^{* * *}$ \\
\hline Challenge & & $0.19^{* *}$ & $0.19^{* * *}$ & $0.17^{* *}$ & & $0.18^{* *}$ & $0.18^{* *}$ & $0.16^{* *}$ & & 0.10 & 0.10 & 0.07 \\
\hline Control & & 0.07 & 0.06 & 0.06 & & 0.06 & 0.04 & 0.05 & & 0.08 & 0.07 & 0.06 \\
\hline Optimism & & & $0.12^{* *}$ & $0.13^{* *}$ & & & $0.19^{* *}$ & $0.19^{* *}$ & & & 0.12 & 0.13 \\
\hline $\begin{array}{c}\text { Positive } \\
\text { affect }\end{array}$ & & & & 0.09 & & & & 0.08 & & & & 0.13 \\
\hline $\begin{array}{l}\text { Negative af- } \\
\text { fect }\end{array}$ & & & & -0.11 & & & & -0.11 & & & & -0.11 \\
\hline $\begin{array}{l}\text { Affect } \\
\text { balance }\end{array}$ & & & & 0.09 & & & & 0.09 & & & & 0.09 \\
\hline $\begin{array}{c}R^{2} \\
\text { (adjusted) }\end{array}$ & 0.07 & 0.36 & 0.37 & 0.40 & 0.04 & 0.36 & 0.39 & 0.41 & -0.01 & 0.21 & 0.26 & 0.30 \\
\hline$\Delta R^{2}$ & 0.07 & 0.29 & 0.01 & 0.03 & 0.04 & 0.32 & 0.03 & 0.02 & 0.00 & 0.22 & 0.05 & 0.04 \\
\hline
\end{tabular}

Table 2. Results of hierarchical regression analyses, standardized regression coefficients. Personality predictors of work engagement among Chinese nurses.

\begin{tabular}{|c|c|c|c|c|c|c|c|c|c|c|c|c|}
\hline & \multicolumn{4}{|c|}{ Dedication } & \multicolumn{4}{|c|}{ Absorption } & \multicolumn{4}{|c|}{ Vigor } \\
\hline & Step 1 & Step 2 & Step 3 & Step 4 & Step 1 & Step 2 & Step 3 & Step 4 & Step 1 & Step 2 & Step 3 & Step 4 \\
\hline $\begin{array}{l}\% \text { of interaction } \\
\text { with patients }\end{array}$ & $-0.21^{* * *}$ & $-0.17^{* *}$ & $-0.17^{* *}$ & $-0.15^{* *}$ & $-0.23^{* *}$ & $-0.19^{* *}$ & $-0.19^{* *}$ & $-0.18^{* *}$ & $-0.29^{* * *}$ & $-0.24^{* * * *}$ & $-0.24^{* * *}$ & $-0.23^{* *}$ \\
\hline Commitment & & $069^{* * * *}$ & $.66^{* * *}$ & $0.61^{* * * *}$ & & $0.62^{* * *}$ & $0.57^{* * *}$ & $0.53^{* * * *}$ & & $0.58^{* * * *}$ & $0.54^{* * *}$ & $0.48^{* * *}$ \\
\hline Challenge & & $019^{* *}$ & $.19^{* *}$ & $0.17^{* *}$ & & $0.18^{* *}$ & $0.18^{* *}$ & $0.16^{* *}$ & & 0.10 & 0.10 & 0.07 \\
\hline Control & & 0.07 & .06 & 0.06 & & 0.06 & 0.04 & 0.05 & & 0.08 & 0.07 & 0.06 \\
\hline Optimism & & & $.12^{* *}$ & $0.13^{* *}$ & & & $0.19^{* *}$ & $0.19^{* *}$ & & & 0.12 & 0.13 \\
\hline Positive affect & & & & 0.09 & & & & 0.08 & & & & 0.13 \\
\hline Negative affect & & & & -0.11 & & & & -0.11 & & & & -0.11 \\
\hline Affect balance & & & & 0.09 & & & & 0.09 & & & & 0.09 \\
\hline$R^{2}$ (adjusted) & 0.07 & 0.36 & 0.37 & 0.40 & .04 & 0.36 & 0.39 & 0.41 & -0.01 & 0.21 & 0.26 & 0.30 \\
\hline$\Delta R^{2}$ & 0.07 & 0.29 & 0.01 & 0.03 & .04 & 0.32 & 0.03 & 0.02 & 0.00 & 0.22 & 0.05 & 0.04 \\
\hline
\end{tabular}

${ }^{*} p<0.05 .{ }^{* *} p<0.01 .{ }^{* * *} p<0.001$.

thesis 1 was only partially confirmed because not all the personality variables included were significant in all the analyses carried out with each dimension of engagement. With regard to Hypothesis 2, the present study is an approximation using a cross-cultural approach that shows that cultural variables are significant, as also mentioned by other authors. Chinese nurses are characterized by higher scores in HP, optimism and positive affect and lower scores in engagement. This suggests that engagement is not an exclusively personal variable, although personality variables are relevant. Engagement also depends on the resources of the organization and on cultural variables [4]. The cultures and the resources provided by organizations in China and Spain are different. In both samples, the variable that was most closely associated with engagement was the commitment dimension of HP, mainly because commitment refers to engagement with life. Nurses who score high in commitment do not shirk their responsibilities, nor do they avoid problems; on the contrary, they deal with them adaptively and positively. For the Chinese nurses, challenge was also a significant predictor, especially to explain the scores in dedication and absorption. When Chinese nurses perceive change as a challenge and not as a stressful situation, their dedi- 
cation and absorption increase. This factor is essential, given the constant changes and developments currently taking place in China, with continuous improvements in the hospitals. In both samples, the role of optimism, positive expectations and the hopes that bad times would pass was also significant and was associated with effective work measures such as positive attitudes, increased job satisfaction and organizational commitment. Lastly, the dimensions of SWB were only significant for the Spanish nurses. This study integrates previous research on engagement, HP, optimism and SWB in nurses from a cross-cultural perspective. The present study is an initial step to understand how the link among these variables strengthens engagement. The findings revealed some applicable and new knowledge related to the effects of HP, optimism and SWB on engagement. These results can contribute to the implementation of hospital policies that, through the development in nurses of HP, optimism and SWB, promote an increase in engagement and the improvement of working environment, relationships with users and co-workers, mutual help, etc. From a cross-cultural perspective, the present study has revealed the main effects of HP, optimism and SWB, indicating that nurses from China and Spain with these personal resources have more engagement. All things considered, the study from this perspective presents more internationally relevant results.

\section{References}

[1] Simpson, M.R. (2009) Engagement at Work: A Review of the Literature. International Journal of Nursing Studies, 46, 1012-1024. http://dx.doi.org/10.1016/j.ijnurstu.2008.05.003

[2] Schaufeli, W.B. and Bakker, A.B. (2004) Job Demands, Job Resources, and Their Relationship with Burnout and Engagement: A Multi-Sample Study. Journal of Organizational Behavior, 25, 293-315. http://dx.doi.org/10.1002/job.248

[3] Hofstede, G. (1980) Culture’s Consequences: International Differences in Work-Related Values. Sage, Newbury Park.

[4] Garrosa, E., Moreno-Jiménez, B., Liang, Y. and González, J.L. (2008) The Relationship between Socio-Demographic Variables, Job Stressors, Burnout, and Hardy Personality in Nurses: An Exploratory Study. International Journal of Nursing Studies, 45, 418-427. http://dx.doi.org/10.1016/j.ijnurstu.2006.09.003 